March 11 issue of Chemistry of Materials, Y. Shirota and co-workers from Osaka University in Japan reported the synthesis and properties of a novel class of highperformance, color-tunable emitting amorphous molecular materials with bipolar characters: 4-dimesitylboryl-N,N-bis(9,9dimethylfluoren-2-yl)aniline (FlAMB-0T); $2-\{4$ - [bis- $(9,9$-dimethylfluoren-2yl)amino]phenyl\}-5-(dimesitylboryl) thiophene (FlAMB-1T); 2-\{4-[bis- $(9,9-$ dimethylfluoren-2-yl)amino]phenyl $\}-2^{\prime}-$ dimesitylboryl-5,5'-bithiophene (FlAMB$2 \mathrm{~T})$; and 5-\{4-[bis $(9,9$-dimethylfluoren-2yl)amino]phenyl -5"-dimesitylboryl2,2':5',2"'-terthiophene (FlAMB-3T).

The molecular design of these materials is based on connecting both electrondonating and electron-accepting moieties (which undergo reversible oxidation and reduction) through a central $\pi$-conjugated system. In other words, the incorporation of the difluorenyl(phenyl)amine and dimesitylboron moieties provide both electron-donating and electron-accepting properties, respectively, and facilitated formation of amorphous glasses due to their nonplanar molecular structures. The central thiophene rings control the highest occupied and lowest unoccupied molecu- lar orbital energy levels, depending on the material's $\pi$-conjugation length. The researchers reported that emission color can be tuned by varying the conjugation length of the thiophene unit.

The researchers reported the fabrication of electroluminescent devices using FlAMB$n \mathrm{~T}$ as emitting or host materials and the development of a high-performance whitelight-emitting electroluminescent device using a bilayer combination of FlAMB-0T and FlAMB-3T.

ANDREI A. ELISEEV

\section{Surface Properties Reversibly Switched Using Electrical Potential}

A research team from the Massachusetts Institute of Technology (MIT), the University of California at Santa Barbara (UCSB), and UC-Berkeley have developed a process that dynamically changes interfacial properties by conformational transitions, or switching, of surface-confined molecules. Unlike other methods that involve chemical reactions, the team has demonstrated the ability to use an active stimulus, such as an electrical potential, to effect such changes without altering the system's environment.

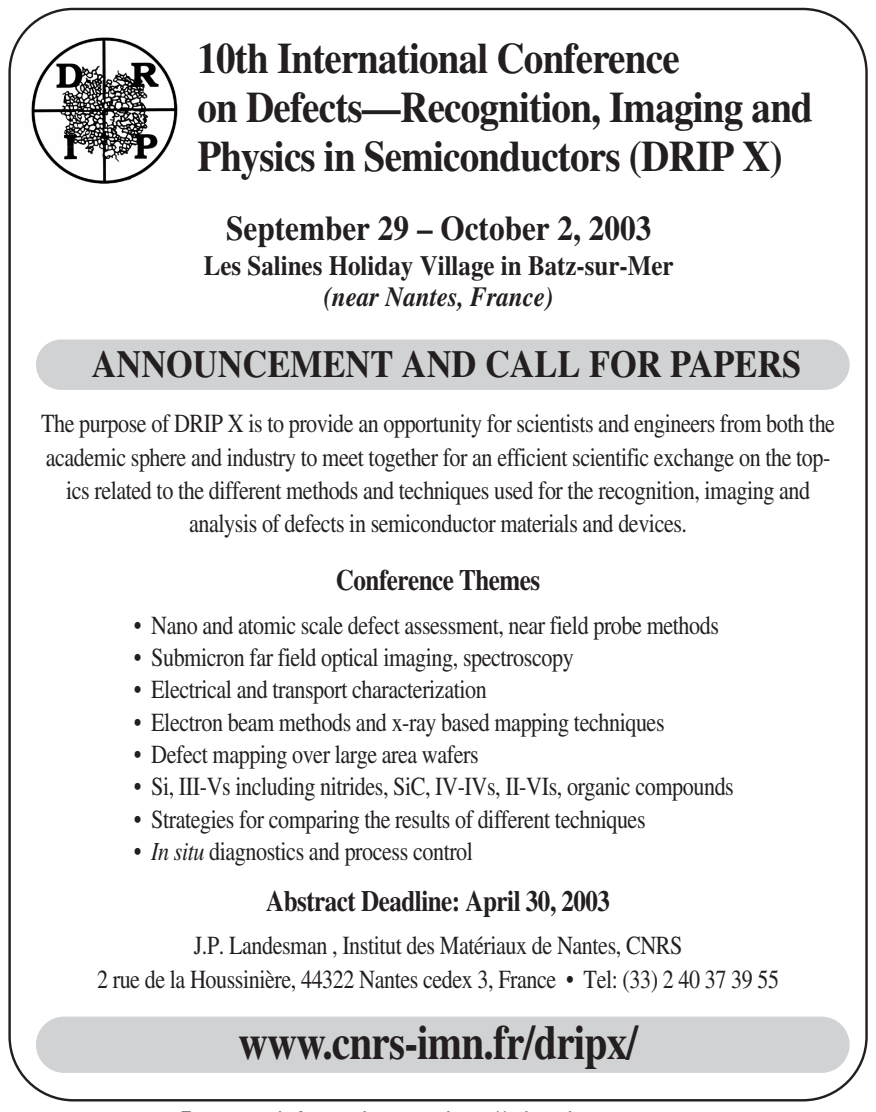

For more information, see http://advertisers.mrs.org
As reported in the January 17 issue of Science, a self-assembled monolayer of (16-mercapto)hexadecanoic acid was prepared on a gold surface. This molecule was chosen because it has a hydrophobic chain and a hydrophilic group on one end; therefore, it may grant different properties to the surface, depending on which part of the molecule is exposed. In order to allow for the switching, the monolayers need to be less dense than conventional self-assembled monolayers; that is, sufficient spatial freedom must be established for each molecule. A monolayer with an optimum spacing between molecules was obtained using a precursor molecule with a globular head group. Hydrolysis of the globular group yielded a low-density monolayer of the molecule of interest.

In the equilibrium state, the molecules organized in a straight fashion on the surface, exposing their hydrophilic end. Joerg Lahann of MIT, Samir Mitragotri of UCSB, and co-workers observed that after applying an electrical potential to the gold surface, the molecules' end groups were attracted toward the charged surface, showing the hydrophilic chains. Sum frequency generation (SFG) spectroscopy was used to analyze the conformational states of the monolayer, since the intensity of the SFG spectrum is affected by the orientation of the molecules. The results show that an electrical potential changes the conformation of the molecules in the monolayer, and that the switching is a reversible process. Contact angles were measured over four switching cycles, and while a large hysteresis was observed, the drop in contact angle was also determined to be reversible. The researchers attributed the observed hysteresis to surface chemical heterogeneity and roughness.

According to the researchers, these findings have important implications for the dynamic control of macroscopic surface properties. The researchers identified applications in microfluidics, microengineering of smart templates, and microfabrication of controlled-release devices.

Maria M. CORTALEZZI

\section{Stable $\alpha$-Phase Nickel Hydroxides Obtained with $10 \%$ Aluminum Substitution}

Alpha-phase nickel hydroxides have recently been investigated in order to increase the performance of rechargeable alkaline batteries. The $\alpha$-nickel hydroxide has a superior electrochemical capacity of $433 \mathrm{mAh} / \mathrm{g}$ theoretical and $350 \mathrm{mAh} / \mathrm{g}$ actual, as compared with $\beta$-nickel hydroxide at $289 \mathrm{mAh} / \mathrm{g}$ theoretical and 273 
Thin Film Solutions

\section{from Experts in}

\section{Deposition Control}

The name Sycon and its logo are synonymous with quality Thin Film Depositions. With Sycon's full line of deposition components and world-class support, you're sure of getting a reliable instrument for your deposition requirement.

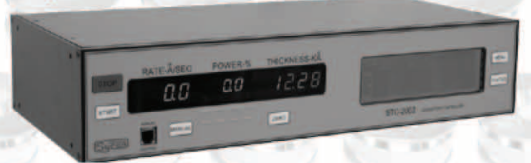

The STC-2002 is the flexible multilayer powerhouse. Expandable to 8 Channels of measurement \& control for very demanding process applications.

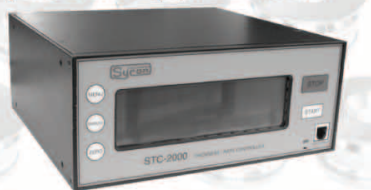

The STC-2000A has the same high-speed precision measurement engine as the 2002. Two measurement channels, handle most deposition applications.

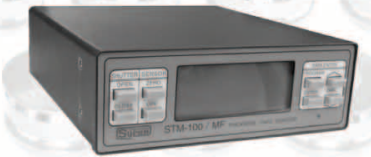

STM-100 / MF, Multi-film monitors. Easy to use, with large back-lit high visibility display. A World standard; 1000's in use.

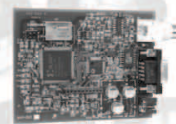

The STM-1, low cost, precision rate/thickness monitor. Single channel monitor with integrated oscillator on a compact pc board.

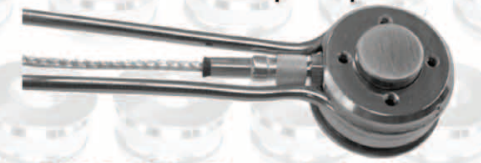

The VSO-100, Sycon's In-Vacuum Sensor / Oscillator. For large system applications.

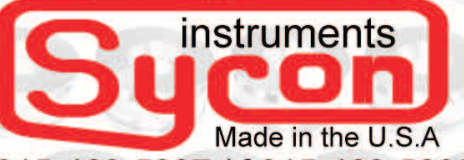

v 315-463-5297 / f 315-463-5298 www.sycon.com

For more information, see http://advertisers.mrs.org
$\mathrm{mAh} / \mathrm{g}$ actual. Utilization of the $\alpha$-nickel hydroxide also avoids the formation of a $\gamma$-phase when overcharged, which results in cell breakdown. Researchers Wei-Kang $\mathrm{Hu}$ and Dag Noréus from the Department of Structural Chemistry at Stockholm University have fabricated pasted electrodes from a stable $\alpha$-phase nickel hydroxide with 10 at.\% Al substitution that display a higher specific capacity and weight energy density than $\beta$-type nickel electrodes.

Because the substitution element ( $\mathrm{Al}$ in this case) does not participate in the electrochemical reaction, the concentration of substituted $\mathrm{Al}$ must be as low as possible. As reported in the February 25 issue of Chemistry of Materials, the Al concentration increases the spacing of the (001) planes in the rhombohedral $\alpha$-phase nickel hydroxide structure, which decreases the tap density (from $1.2 \mathrm{~g} / \mathrm{cm}^{3}$ to $1.0 \mathrm{~g} / \mathrm{cm}^{3}$ as the $\mathrm{Al}$ content increases from 10 at.\% to 30 at.\%), leading to lower energy density with increased Al content. Experiments showed that samples with 10 at. \% Al showed sufficient stability after aging in $6.0 \mathrm{M} \mathrm{KOH}$ for 48 days at room temperature with no $\beta$ phase observed. In addition, no $\beta$ phase was observed after 300 charge-discharge cycles in the NiMH battery cells.

AA-size $\alpha$-type and $\beta$-type NiMH cells were constructed for material comparison. The results showed that the $\beta$-type nickel electrode has a capacity of $1500 \mathrm{mAh}$ at a $0.2 \mathrm{C}$ rate, while the $\alpha$-type electrode displayed a lower capacity near $1100 \mathrm{mAh}$, due to its lower volumetric energy density. As the $\beta$-type nickel electrodes are already near their theoretical maximum, it is hoped that the results of this study, which show functioning $\alpha$-type nickel pasted electrodes, will pave the way for further improvements in the weight and volumetric energy densities of these materials, leading to increased cell performance.

KYLE BRINKMAN

\section{FOR MORE RESEARCI NEWS ON MATERIAIS SCIENCE ... \\ ... access the Materials Research Society Web site: \\ www.mrs.org/gateway/ matl_news.html}

\section{Pan-American Advanced Studies Institute (PASI) on Materials for Energy Conversion and Environmental Protection \\ Rio de Janeiro, Brazil • October 20-29, 2003}

Topics: - Fuel Cell Principles, Design, and Applications (Energy Conversion)

- Catalytic Materials for Emissions Reduction (Environmental Protection)

- Policy-making and Funding Resource Management

Program Highlights: - Distinguished Pan-American Lecturers from Academia,
- Government and Industry
- Policy and Technical Sessions
- International Networking
- Interdisciplinary, Intercultural Working Groups and
Panel Discussions
- Based on mutual interests, student groups will identify
potential collaborations and prepare joint proposals to
obtain the necessary funding.

The PASI will generate real partnerships among Pan-American scientists and provide the tools and direction needed to sustain their future collaborations.

Advanced graduate and postgraduate students may download an application from our website: http://www.materialsworld.net/PASI/.

Applications must be received by June 1, 2003.

Co-organized by the Materials Research Institute (Northwestern University) and the Materials Research Society of Brazil. Co-sponsored by the US Department of Energy, the National Science Foundation, \& the US Army Research Office 